\title{
Diagnosing snapping popliteus tendon is not a snap in lateral knee symptoms
}

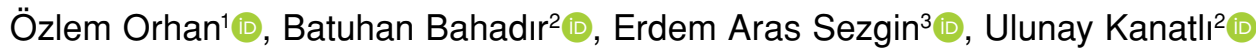 \\ 'Department of Orthopedics and Traumatology, Harran University Faculty of Medicine, Şanlıurfa, Turkey \\ ${ }^{2}$ Department of Orthopedics and Traumatology, Gazi University Faculty of Medicine, Ankara, Turkey \\ ${ }^{3}$ Department of Orthopedics and Traumatology, Aksaray University Faculty of Medicine, Aksaray, Turkey
}

Although snapping sensation that causes pain in the lateral aspect of the knee is a common symptom, snapping of the popliteus tendon (PT) over the lateral femoral condyle rarely presents as the cause. Anatomy, function, and ruptures of the PT have been studied extensively and are well documented in the literature ${ }^{[1-3]}$ However, clinical and radiological diagnosis of snapping is still challenging, resulting in an increased risk of incorrect diagnosis and eventually inadequate treatment. It can be easily missed among other symptoms of the lateral knee, such as iliotibial band friction syndrome, lateral meniscus tear, discoid lateral meniscus, cartilage injury or loose bodies in the knee joint.

\section{ABSTRACT}

Painful snapping located at lateral aspect of the knee is common; however, snapping popliteus tendon syndrome (SPTS) rarely presents as the cause and may be misdiagnosed even by the most experienced surgeons. A 36-year-old male patient was admitted with lateral knee pain and snapping sensation for two years. Snapping was palpated at the lateral knee when flexed at $30^{\circ}$ and the patient described intense pain beyond $80^{\circ}$. Arthroscopy revealed thickening of intra-articular portion of the popliteus tendon (PT). Surrounding synovium had extensive inflammation. During flexion, PT snapped back and forth into the popliteal sulcus. The inflamed tissue was debrided with no additional intervention to the PT. Postoperative physical therapy was recommended. The patient was relieved of his symptoms by the end of the first year. In conclusion, surgeons should be aware of SPTS during evaluation of the lateral compartment for optimal management of patients presenting with lateral knee pain and snapping sensation.

Keywords: Arthroscopy, iliotibial band syndrome, knee, lateral, popliteus tendon, snapping.

Herein, we present a rare case of snapping PT syndrome (SPTS) and discuss clinical presentation, early interventions, and arthroscopic diagnosis in the light of literature data.

\section{CASE REPORT}

A 36-year-old male farmer presented to our outpatient clinic with a two-year history of activity-related pain and snapping sensation on the lateral aspect of his left knee. He had no history of trauma. He underwent an arthroscopic lateral partial meniscectomy 15 months prior in an external center with a preliminary diagnosis of lateral meniscus tear. However, this procedure failed to address patient's symptoms of painful 

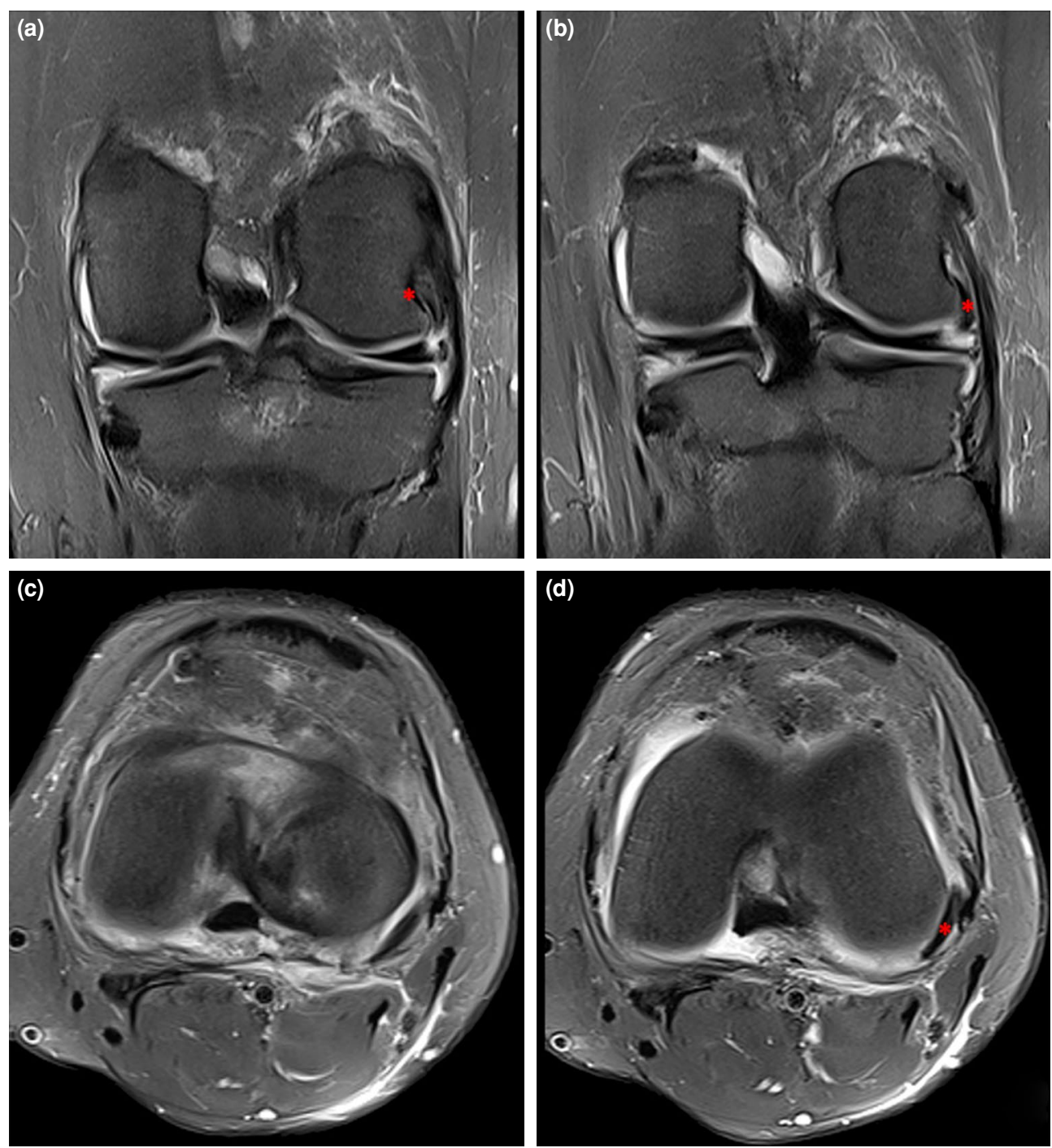

Figure 1. Magnetic resonance imaging of the knee. (a) Popliteus tendon origin in the coronal plane is marked with a red asterisk. (b) Popliteus tendon's relation to the lateral femoral condyle in the coronal plane. Red asterisk represents the body of the tendon Figures 1c and 1d. Horizontal plane images showing the relation of popliteus tendon (asterisk) to tibia and femur.

lateral snapping and locking sensation occurring, particularly while standing up from a squatted position. The patient was unable to work due to these symptoms. His physical examination revealed normal gait and Ober's test was negative. There were no findings indicating a ligament or meniscus injury. Snapping was palpated on the lateral aspect of left knee during $30^{\circ}$ to $40^{\circ}$ of passive flexion and flexion over $80^{\circ}$ triggered intense pain. Direct radiographic images were normal. Magnetic resonance imaging (MRI) revealed expected findings secondary to partial meniscectomy and non-specific inflammation on the lateral knee (Figure 1a-d). Considering clinical and radiological findings, the patient was offered diagnostic arthroscopy without a definitive preoperative diagnosis. The patient was informed that data from the case would be submitted for publication and gave their consent.

Arthroscopy revealed lateral meniscus remnant was healthy and there were no signs of meniscus tear, articular cartilage injury, and loose bodies. Upon viewing the lateral compartment, intraarticular portion of the PT was seen to be thickened and clearly apparent. Thus, PT was examined further (Figure 2a, b). Tendon was fibrotic when examined with an arthroscopic hook. Popliteus tenosynovitis was observed, and the surrounding synovium had extensive inflammation (Figure 2c, d) 

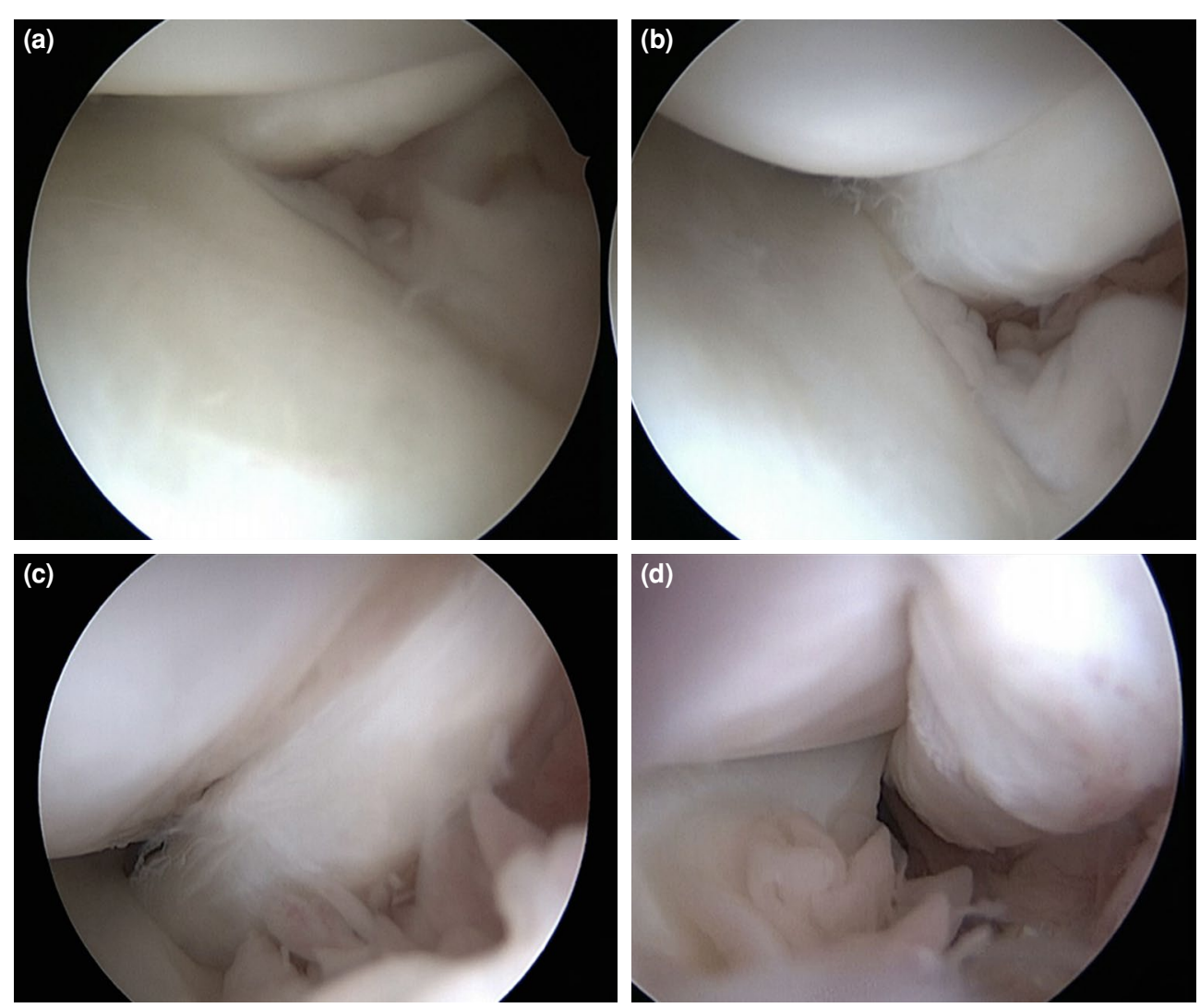

Figure 2. Arthroscopic view of the popliteus tendon from the anterolateral portal. (a-c) Anterior subluxation out of the sulcus causes "more tendon" appearance. Fraying and inflammation on the tendon indicates friction. (d) Top-down view of the tendon from the anterolateral portal. Extensive inflammation of the tendon and surrounding synovial tissue is observed.

(see additional Video 1). During passive range of motion, intra-articular portion of the PT subluxated anteriorly and snapped back and forth into the popliteal sulcus

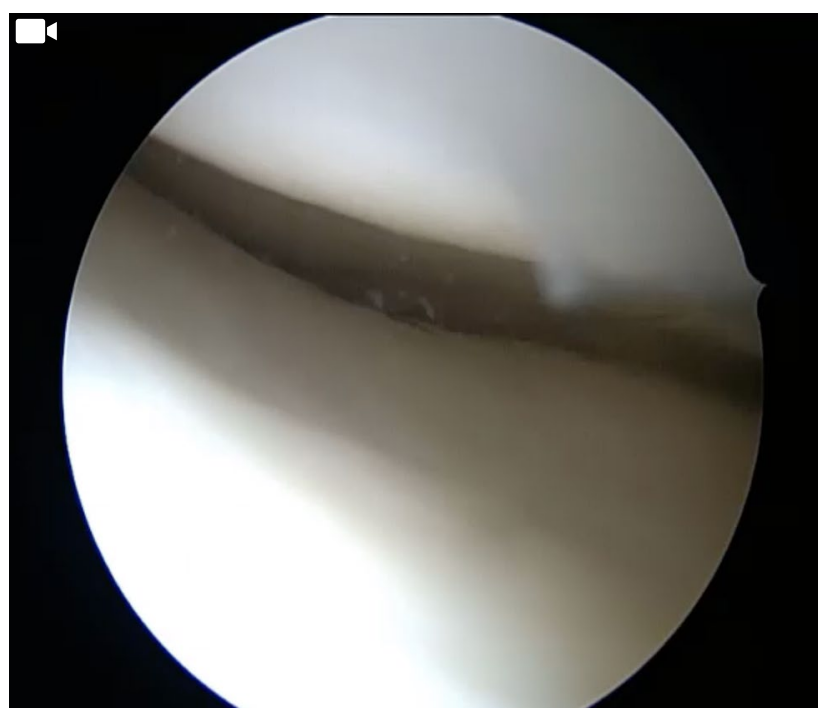

Video 1. Click image to view online. Video from the arthroscopic procedure showing a subluxated popliteal tendon with surrounding tenosynovitis and extensive synovial inflammation. on the lateral femoral condyle. Considering patient's activity level, no other interventions related to PT was applied, except for debridement of the inflamed tissue. There were no complications during the perioperative period. Non-specific physical therapy was initiated rapidly and he was recommended to rest and lower his workload. Twelve months after surgery, the patient had significantly reduced pain with a normal range of motion. Although he had persistent snapping sensation, it did not alter any sort of pain perception. Squatting was without trouble and he returned to work.

\section{DISCUSSION}

Due to challenges in diagnosis, SPTS may be misdiagnosed even by the most experienced of orthopedic surgeons. Consequently, these patients may receive inadequate treatment for other etiologies in lateral knee pain, such as iliotibial band friction syndrome and lateral meniscus injury. Our case perfectly demonstrates this difficulty, who was treated with arthroscopic partial lateral meniscectomy earlier. Preoperative diagnosis was 
not clear upon admission to our clinic, as well. However, when the preoperative MRI sections were re-evaluated after arthroscopy, a thickened and subluxated PT was noted.

Snapping PT was first described by Crites et al. ${ }^{[4]}$ in 1998. Later, several others also reported their experience regarding the diagnosis and treatment of this rather "easy-to-miss" syndrome. A tight tendon or a shallow femoral sulcus were suggested as potential cause behind snapping, although no clear cause has been identified. ${ }^{[5]}$ It appears that, if diagnosed correctly, pain and disability caused by snapping popliteus can be successfully addressed with conservative treatment which includes rest from aggravating activities, non-steroidal anti-inflammatory drugs and physical therapy. ${ }^{[5-7]}$ However, if this yields no benefit in reduction of symptoms, surgical options should be considered. Removal of the prominent edge of the popliteal sulcus with an osteotome, tenodesis by anchoring the PT to the proximal half of the lateral collateral ligament or to the femoral sulcus, tubercle smoothing and removing osteophyte causing impingement and removal of symptomatic cyamella in the PT have all been described as open techniques with satisfactory outcomes. ${ }^{[4,6-10]}$ Mariani et al. ${ }^{[5]}$ described the arthroscopic findings and they were the first to describe arthroscopy as a treatment option. They defined the arthroscopic finding as "more tendon", indicating the portion of the tendon near its femoral insertion that is usually hidden by the condyle being clearly evident. One of their three patients was treated with arthroscopic popliteal sulcus deepening and tenodesis using a bioabsorbable suture anchor inserted into the sulcus. One of the other two was treated conservatively and other underwent open tenodesis, all with successful outcomes. Our patient was relieved of his symptoms by the end of the first year after the operation; however, follow-up is recommended for more years and, if deemed necessary, an arthroscopic tenodesis and deepening of popliteal sulcus may be planned.
In conclusion, surgeons should be aware of SPTS during evaluation of the lateral compartment for optimal management of patients presenting with lateral knee pain and snapping sensation. Successful outcomes with surgical interventions can be achieved or, in some cases, physical therapy alone is sufficient. However, the definitive diagnosis should be made first, usually with arthroscopy.

\section{Declaration of conflicting interests}

The authors declared no conflicts of interest with respect to the authorship and/or publication of this article.

\section{Funding}

The authors received no financial support for the research and/or authorship of this article.

\section{REFERENCES}

1. Mann RA, Hagy JL. The popliteus muscle. J Bone Joint Surg [Am] 1977;59:924-7.

2. Chahla J, James EW, Cinque ME, LaPrade RF. Midterm outcomes following anatomic-based popliteus tendon reconstructions. Knee Surg Sports Traumatol Arthrosc 2018;26:812-8.

3. Fineberg MS, Duquin TR, Axelrod JR. Arthroscopic visualization of the popliteus tendon. Arthroscopy 2008;24:174-7.

4. Crites BM, Lohnes J, Garrett WE Jr. Snapping popliteal tendon as a source of lateral knee pain. Scand J Med Sci Sports 1998;8:243-4.

5. Mariani PP, Mauro CS, Margheritini F. Arthroscopic diagnosis of the snapping popliteus tendon. Arthroscopy 2005;21:888-92.

6. Cooper DE. Snapping popliteus tendon syndrome. A cause of mechanical knee popping in athletes. Am J Sports Med 1999;27:671-4.

7. Krause DA, Stuart MJ. Snapping popliteus tendon in a 21-year-old female. J Orthop Sports Phys Ther 2008;38:191-5.

8. Gaine WJ, Mohammed A. Osteophyte impingement of the popliteus tendon as a cause of lateral knee joint pain. Knee 2002;9:249-52.

9. McAllister DR, Parker RD. Bilateral subluxating popliteus tendons. A case report. Am J Sports Med 1999;27:376-9.

10. Su S, Lu Y, Chen Y, Li Z. A symptomatic cyamella in the popliteus tendon causing snapping knee: A case report and literature review. BMC Musculoskelet Disord 2019;20:495. 